University of Wollongong

Research Online

Faculty of Social Sciences - Papers (Archive) Faculty of Arts, Social Sciences \& Humanities

2012

Testing relationships: Ethical arguments for screening for type 2 diabetes mellitus with $\mathrm{HbA} 1 \mathrm{C}$

Christopher J. Degeling

University of Wollongong, degeling@uow.edu.au

Melanie Rock

University of Calgary

Wendy Rogers

Macquarie University, wendy.rogers@scmp.mq.edu.au

Follow this and additional works at: https://ro.uow.edu.au/sspapers

Part of the Education Commons, and the Social and Behavioral Sciences Commons

Research Online is the open access institutional repository for the University of Wollongong. For further information contact the UOW Library: research-pubs@uow.edu.au 


\title{
Testing relationships: Ethical arguments for screening for type 2 diabetes mellitus with $\mathrm{HbA} 1 \mathrm{C}$
}

\author{
Abstract \\ Since the 1990s, glycated haemoglobin (HbA1C) has been the gold standard for monitoring glycaemic \\ control in people diagnosed as having either type 1 diabetes mellitus (T1DM) or type 2 diabetes mellitus \\ (T2DM). Discussions are underway about diagnosing diabetes mellitus on the basis of HbA1C titres and \\ using $\mathrm{HbA} 1 \mathrm{C}$ tests to screen for T2DM. These discussions have focused on the relative benefits for \\ individual patients, with some attention directed towards reduced costs to healthcare systems and \\ benefits to society. We argue that there are strong ethical reasons for adopting $\mathrm{HbA} 1 \mathrm{C}$-based diagnosis \\ and T2DM screening that have not yet been articulated. The rationale includes the differential impact of \\ $\mathrm{HbA} 1 \mathrm{C}$-based diabetic testing on disadvantaged groups, and what we are beginning to learn about $\mathrm{HbA} 1 \mathrm{C}$ \\ vis-à-vis population health. Although it is arguable that screening must primarily benefit the individual, \\ using $\mathrm{HbA} 1 \mathrm{C}$ to diagnose and screen for T2DM may promote a more just distribution of health resources \\ and lead to advances in investigating, monitoring and tackling the social determinants of health.

\section{Disciplines \\ Education | Social and Behavioral Sciences}

\section{Publication Details} \\ Degeling, C., Rock, M. \& Rogers, W. A. (2012). Testing relationships: Ethical arguments for screening for \\ type 2 diabetes mellitus with HbA1C. Journal of Medical Ethics, 38 (3), 180-183.
}


This is a pre-copyedited, author-produced PDF of an article accepted for publication in J Med Ethics following peer review. The definitive publisher-authenticated version [Degeling C, Rock M, \& Rogers W. (2012) Testing relationships: ethical arguments for screening with HbA1C. Journal of Medical Ethics. 38 (2) 180-83.] is available online at http://ime.bmj.com/content/38/3/180.short

\title{
Testing relationships: ethical arguments for screening for type 2 diabetes mellitus with $\mathrm{HbA1C}$
}

\author{
Chris Degeling, Melanie Rock, Wendy Rogers (2012)
}

\begin{abstract}
Since the 1990s, glycated haemoglobin (HbA1C) has been the gold standard for monitoring glycaemic control in people diagnosed as having either type 1 diabetes mellitus (T1DM) or type 2 diabetes mellitus (T2DM). Discussions are underway about diagnosing diabetes mellitus on the basis of $\mathrm{HbA} 1 \mathrm{C}$ titres and using $\mathrm{HbA} 1 \mathrm{C}$ tests to screen for T2DM. These discussions have focused on the relative benefits for individual patients, with some attention directed towards reduced costs to healthcare systems and benefits to society. We argue that there are strong ethical reasons for adopting $\mathrm{HbA} 1 \mathrm{C}$-based diagnosis and T2DM screening that have not yet been articulated. The rationale includes the differential impact of $\mathrm{HbA} 1 \mathrm{C}$-based diabetic testing on disadvantaged groups, and what we are beginning to learn about $\mathrm{HbA} 1 \mathrm{C}$ vis-à-vis population health. Although it is arguable that screening must primarily benefit the individual, using $\mathrm{HbA} 1 \mathrm{C}$ to diagnose and screen for T2DM may promote a more just distribution of health resources and lead to advances in investigating, monitoring and tackling the social determinants of health.
\end{abstract}

\section{Introduction}

The rising incidence of type 2 diabetes mellitus (T2DM) is a pressing global health concern. The need to identify individuals at elevated risk of diabetic complications more reliably and earlier has prompted discussions as to the costs and benefits of changing the diagnostic criteria from a glucosebased measure to glycated haemoglobin ( $\mathrm{HbA1C}$ ) (box 1), with a suggested threshold for diabetes of 6.5\% HbA1C.1 While HbA1C measures could also be used to confirm type 1 diabetes mellitus (T1DM), since T1DM usually presents acutely, the practical and ethical issues associated with $\mathrm{HbA1C}$ based diagnosis apply chiefly to T2DM. The primary advantage of HbA1C-based tests is that they measure an individual's exposure to hyperglycaemic states over a period of weeks, rather than hours, which can facilitate the identification of low levels of persistent hyperglycaemia. The other key reason to change diagnostic techniques for diabetes is the stable relationship between an individual's HbA1C titres and their risk of macrovascular disease. Currently, fasting plasma glucose levels (FPG) for diagnosing diabetes are set at $7.0 \mathrm{mmol} / \mathrm{l}$ because there is a sharp inflexion at this point for the risk of microvascular disease (renal, vision, etc.). It has not, however, been possible to set a diagnostic criterion based on FPG to reflect risk of macrovascular disease, because an individual's risk of cardiovascular pathologies rises gradually relative to FPG. Consequently, current methods of diagnosing diabetes do little to mitigate the risks of macrovascular diseases in individual cases. 2 When examined from both primary care and population health perspectives, the lag 
between onset of cellular dysfunction and glucose-based clinical diagnosis (estimated at 7 years, on average) is now thought to have a major impact on prognoses for patients with T2DM. Because $\mathrm{HbA} 1 \mathrm{C}$ levels can be used to predict both microvascular disease and the risks of fatal and non-fatal macrovascular events in pre-diabetic and diagnosed cases of T2DM, the American Diabetic Association has recently recommended that $\mathrm{HbA} 1 \mathrm{C}$ levels should be used to define and diagnose diabetic diseases.1

Box 1: Glycated haemoglobin (HbA1C), diabetes mellitus, cardiovascular disease and population health

$\mathrm{HbA} 1 \mathrm{C}$ is a blood-borne glycoprotein that forms through a direct reaction between plasma glucose and haemoglobin. Foundational trials conducted in the 1990s established $\mathrm{HbA} 1 \mathrm{C}$ assays as a reliable index of glycaemic control in individuals with diabetes.3 4 More recent population-based studies confirm that $\mathrm{HbA1C}$ is also a reliable marker of cardiovascular risk in both non-diabetic and diabetic populations, although the causal role of glucose in cardiovascular disease is as yet unclear. 5 Social epidemiologists have used $\mathrm{HbA1C}$ as a biological measure to compare with self-rated health. Studies have consistently found raised $\mathrm{HbA1C}$ levels associated with increased exposure to stressors in nondiabetic individuals and that there is an inverse and independent relationship between raised $\mathrm{HbA} 1 \mathrm{C}$ and socioeconomic status and social disadvantage. 6 Hence $\mathrm{HbA} 1 \mathrm{C}$ is a stable biomarker of an individual's exposure to hyperglycaemic states and cardiometabolic risks, even in patients without measurable abnormalities in glucose metabolism or who are below the threshold for clinical T2DM. The move to HbA1C-based diagnosis is pragmatic. The aim is to identify at-risk individuals earlier, well before they have clinically evident glucose dysregulation and an elevated risk of diabetic complications.

\section{Current practices and debates surrounding T2DM screening, diagnosis and prevention}

Diagnosis and screening are distinct types of clinical practice. Diagnosis is a process of identifying potential abnormalities in an affected individual seeking healthcare, whereas screening is used to identify members of a population who have a specific condition, or are at heightened risk of developing it, but who are not specifically seeking treatment. Diagnosis generally presupposes some form of intervention, whereas screening may focus the attention of individuals, care providers and policy makers on preventive actions. Clinical screening for T2DM has generally only been undertaken on an ad hoc and opportunistic basis in most developed nations. As evidence continues to emerge that earlier diagnosis and treatment of hyperglycaemia and related metabolic abnormalities is beneficial, 89 a growing number of professional organisations are advocating targeted screening for T2DM in high-risk populations.10 Past attempts to screen for these conditions have been haphazard, relied on a variety of different tests and cut-offs, and have had variable success. Although the utility of population-level screening for T2DM remains controversial, the emerging consensus is that $\mathrm{HbA1C}$ may be a better screening tool than the alternatives.11 12 Systematic use of HbA1C assays may prove to be a powerful secondary prevention measure, as the earlier identification of at-risk and pre-diabetic individuals provides those affected and their primary care providers with a better chance of avoiding the onset, risks and burdens of diabetic complications (assuming that relevant services are available).

Past reluctance to adopt $\mathrm{HbA} 1 \mathrm{C}$ as a diagnostic criterion for T2DM reflected legitimate concerns about technological standardisation, which have now been met. Like any test, $\mathrm{HbA} 1 \mathrm{C}$ assays have limitations, but in terms of stability, accuracy and precision, they are now considered the equal of 
standard glucose-based testing protocols.1 13 As well as providing a longitudinal measure of hyperglycaemia and macrovascular risks, there are practical advantages to HbA1C testing. HbA1C testing procedures are convenient. They do not require the patient to fast, and the assay can readily be bundled with other tests, unlike oral glucose tolerance tests (OGTT) and FPG measurements. $\mathrm{HbA} 1 \mathrm{C}$ is an accurate test for hyperglycaemia and can be easily and immediately administered in primary care settings. In contrast, FPG and OGTTs are intrusive, can be difficult to interpret, and rely on high levels of patient compliance. FPG and OGTT also result in substantial under-diagnosis; epidemiological surveys consistently estimate this to be as high as $30-50 \%$ of all cases.11

\section{HbA1C and social epidemiology}

As well as being a direct measure of glycaemic levels, $\mathrm{HbA} 1 \mathrm{C}$ titres have been used as a measure of population health unrelated to T2DM screening. Such research sought to investigate social inequalities in disease prevalence and outcomes associated with social gradients, which were otherwise unexplained by classic behavioural dimensions of health risk exposure. Social epidemiologists used $\mathrm{HbA1C}$ as a biomarker to compare with individuals' self-rated health, reflecting relative intensity and health effects of the social environment and psychosocial stress in populations and subpopulations. Studies have consistently found raised $\mathrm{HbA1C}$ levels associated with increased exposure to stressors in non-diabetic individuals, and identified an inverse and independent relationship between raised $\mathrm{HbA} 1 \mathrm{C}$ and socioeconomic status and social disadvantage. 67 Because of this association, aggregated databases of $\mathrm{HbA1C}$ stand to provide population risk measures, and vital insights into how the health of entire populations is influenced by social determinants, including emotional well-being, social networks and characteristics of where people live. The potential to use a biological measure such as $\mathrm{HbA1C}$ to assess the relative health and exposure to stressors in different populations has important implications in health policy formation and for the allocation of scarce resources.

\section{Ethical grounds for adopting $\mathrm{HbA1C}$ as the diagnostic screening test for T2DM}

While the evidentiary and practical advantages of using $\mathrm{HbA1C}$ as a diagnostic screen for T2DM and as a measure of population health are matters for careful and considered discussion, we argue that the test has ethical advantages over existing clinical practices. These advantages occur for individuals in primary care settings, and at the level of research, planning and intervention to improve the overall health of populations (Box 2).

\section{Box 2: A case study}

The value of these kinds of data is illustrated by recent research conducted with black military veterans living with T2DM in Philadelphia, USA. The findings from this research showed that patients who perceived their neighbourhoods as places where people work together for improvements tended to have lower HbA1C scores than those who did not perceive their neighbourhoods in this way. 32 As perceptions of neighbourhoods are modifiable, this study suggests that interventions aimed at improving perceptions by fostering experiences of community inclusion and social support could prove to be important for preventing the onset of T2DM, as well as of related health problems.

\section{Screening and diagnosis in primary care}

Screening a population for a disease is not necessarily a benign activity; it can cause harms as well as provide benefits. Aside from the risks of false positives and unnecessary interventions, the mere 
invitation to undergo a medical procedure can change people's image of themselves, thereby challenging and even violating their personal integrity.14 There is some ethical consensus around the view that the benefits of a screening programme must accrue to those individuals who take part, rather than to third parties. Participation in a screening programme should reflect an autonomous choice, informed by a clear understanding of the possible individual benefits and harms.15 Recent research has largely allayed concerns that informed consent deters participation, or causes unnecessary anxiety or an unwarranted sense of insecurity for those who choose to be screened.16 17 In relation to screening for T2DM, early detection seems to be beneficial for the individual, especially if microvascular risks, macrovascular risks and metabolic abnormalities can be mitigated by lifestyle changes. Thus the potential harms of offering screening for T2DM to a targeted population appear to be outweighed by the benefits. Notwithstanding the limits of autonomous choice as an ethical framework,18 there remains ethical concern about how best to provide disadvantaged and 'at-risk' groups access to screening programmes.19 20

$\mathrm{HbA1C}$-based screening may be superior to glucose tests for addressing this concern. The test is more convenient for the patient and far simpler to understand than the complicated protocols and confusing classifications that surround glucose-based tests and diagnostic categories. This leads to two consequences. First, as the costs and benefits of diabetes screening with $\mathrm{HbA1C}$ are more easily explained to individual patients than are the alternatives (i.e., FPG and OGTT protocols), there is an increased likelihood that those being tested will give valid informed consent for screening procedures. Second, $\mathrm{HbA1C}$, as a simpler testing regimen than the alternatives, has the potential to make diagnostic procedures-whether it is a screen for an asymptomatic individual or a test to confirm the T2DM diagnosis-more accessible to socially disadvantaged groups and to those unable to comply readily with complicated test regimens. $16 \mathrm{HbA} 1 \mathrm{C}$ assays facilitate opportunistic screening, thereby avoiding a loss to follow-up of those who do not return for confirmatory diagnostic tests (as is required for FPG and OGTT protocols), but who would be willing and able to fill a prescription, to modify their diets, or to become more physically active based on diagnostic test results delivered in a timely fashion. Moreover, even when individuals do not benefit from continuity of care or from medical technologies such as pharmaceuticals to lower blood sugar levels, diagnostic and screening data derived can enable population surveillance and related interventions (eg, improving access to healthcare among disadvantaged social groups).

Adopting $\mathrm{HbA} 1 \mathrm{C}$ as the diagnostic criterion for diabetes may promote a better balance between the core values and principles that underpin current conceptions of ethical clinical practice. Early identification of those at risk provides scope for the timely formation of interpersonal providerpatient relationships, attention to social processes, and concern for life plans. All these features are now considered to be central to effective, efficacious and respectful care of people with long-term conditions.21 $22 \mathrm{HbA1C}$ screening may more easily meet the requirements of non-maleficence and beneficence compared with alternatives, as more accurate testing and earlier diagnosis is likely to promote more favourable outcomes for high-risk individuals. The only caveat to this claim is that the lifestyle risks that promote T2DM must be modifiable. If it turns out that disadvantaged individuals are unable to address the drivers of macrovascular diseases, then the increasing incidence of these conditions among these groups could serve to highlight the need for structural reforms and population-level interventions. 


\section{Extending diabetic research to disadvantaged populations}

Another potential ethical benefit of $\mathrm{HbA1C}$ screening is that it may promote greater justice in research by facilitating the inclusion of socially disadvantaged groups in evaluating the effectiveness of approaches to diabetic management relevant to their situations.

Disadvantaged groups are at highest risk for incidence of T2DM, for related complications, and for comorbidities. Members of these groups often have low health literacy, experience low levels of provider access, face significant psychosocial stressors, and struggle to manage the extra burdens imposed by diabetes treatment.23 These groups are far less likely to have access to appropriate and effective interventions for pre-diabetes and T2DM,24 and generally less likely to participate in and benefit from relevant T2DM research, which currently focuses on pharmacological interventions and modifying the behavioural patterns of individual patients. While emerging evidence indicates that earlier intervention can provide for sustained prevention of T2DM,25 the challenge is to translate these findings to the 'real' world.26 To the extent that lifestyle risks are modifiable, then there is a strong likelihood that disadvantaged populations would disproportionately benefit, and do better with earlier diagnosis.

Yet the research evidence that informs these types of individually focused clinical interventions may not reflect the circumstances and needs of disadvantaged groups. This kind of mismatch can occur through a lack of representation in research design, and through exclusion criteria such as presence of comorbidities and participant availability, leading to a lack of evidence that is applicable to those excluded from studies. Given the importance of research evidence in informing resource allocation decisions and policy development, the lack of evidence as to effectiveness in disadvantaged populations is a serious issue that compromises access to care and health equity. 27 Using $\mathrm{HbA} 1 \mathrm{C}$ as a research tool could facilitate the inclusion of disadvantaged groups in relevant studies, thereby avoiding these potential harms. Moreover, it is likely that making these services accessible to those who need them most will benefit the whole of society, through development of more equitable service models and lower rates of morbidity and mortality. 28

Beyond their facility as a tool to evaluate interventions in primary care settings and members of hard-to-reach populations, $\mathrm{HbA1C}$ titres are also a useful gauge of population health, including the effects of exposure to environmental stressors. Modest increases in the level of $\mathrm{HbA} 1 \mathrm{C}$ have been correlated with key measures of socioeconomic status and job stress, 6 potentially contributing to increased risk of heart disease and other morbidities in non-diabetic populations. The systematic collection of $\mathrm{HbA1C}$ information to create population health databases has potential for opening up new ways of investigating the complex pathways through which socioeconomic and structural factors impact on health. Although our understanding of the mechanisms through which social environments affect health remains limited and partial, the available evidence suggests that social relationships and social dimensions of environments may have significant effects on the success or failure of attempts to address burdens of chronic disease in diabetic and non-diabetic individuals. The link between $\mathrm{HbA} 1 \mathrm{C}$ levels and macrovascular risks is potentially of great significance because most people with diabetes, and many without diabetes, will die from macrovascular disease. 5 At the same time, microvascular risks such as vision loss and kidney failure are often dreaded more than macrovascular risks by individuals with diabetes.29 Elevated $\mathrm{HbA1C}$ levels correlate with both macrovascular and microvascular risks, and also appear to have value vis-à-vis measuring the impact of exposure to stressors. For these reasons, using HbA1C to diagnose and screen for T2DM could effectively address the ethical tensions between population- and individual-level priorities in screening. 
If $\mathrm{HbA1C}$ proves to be a reliable research tool investigating the links between health and the social, economic and cultural circumstances of disadvantage, there are potential benefits, including novel intervention methods and increased opportunities for understanding and addressing the effects of disadvantage on health. The creation of population health databases of $\mathrm{HbA1C}$ could further these aims.30 For example, a population map identifying areas of raised $\mathrm{HbA1C}$ could inform social interventions, again with greatest benefit to the worst-off.31 HbA1C-based testing may provide a greater differential benefit for disadvantaged groups through a timelier diagnosis of insulin intolerance; in addition, because $\mathrm{HbA} 1 \mathrm{C}$ also reflects the effects of other macrovascular risk factors, the systematic collection of these data may have a differential impact on the prioritisation of health outcomes at the level of populations. If this then leads to the greatest benefits accruing to the most disadvantaged, it is possible that $\mathrm{HbA} 1 \mathrm{C}$ may promote greater justice in healthcare.

\section{The case for Hb1AC testing and evidence-based social medicine}

Thirty years ago, Geoffrey Rose argued that medicine has a duty of care not only to individual patients but also to the communities and populations to which we all belong. 33 Social relationships and environments appear to exert direct influences on major causes of mortality, including from cardiovascular disease.34 Therefore, non-medical determinants of population health deserve our attention when discussing the relative merits of diagnostic tests for T2DM. While we must ensure that targeted individuals are the beneficiaries of screening programmes, not all of these benefits need be direct. We should also consider the potential for benefits to the health of populations, as, after all, individuals stand to benefit from improvements in population health.

Addressing the social determinants of health and the associated health gradient is a matter of justice; however, it is difficult for healthcare providers to address broader issues of justice, as their focus is on individual patients presenting for care. Nonetheless, $\mathrm{HbA1C}$ data may be suited to providing the type of evidence base and evaluative framework necessary to broaden the focus of macrovascular disease prevention and treatment interventions beyond clinical settings.

With the routine availability of $\mathrm{HbA} 1 \mathrm{C}$ administrative data derived from screening for T2DM as well as from monitoring people diagnosed with T2DM, it would be possible to measure longitudinally the overall population health impact of social interventions such as community development initiatives and to compare the cost-effectiveness of social interventions with pharmaceutical treatments. Given that the net impact of patient self-management on the health gradient is likely to be very small,35 inequities in outcomes for T2DM and macrovascular disease are unlikely to lessen significantly, unless these new forms of evidence can be generated, evaluated and integrated into established systems. 23

\section{Conclusion}

HbA1C-based testing for T2DM has significant practical and ethical benefits over existing diagnostic and screening protocols. $\mathrm{HbA1C}$ testing for T2DM should improve access to care for disadvantaged populations - who are at heightened risk of and over-represented among people with T2DM. Any step that leads to greater inclusion of these groups is likely to promote greater justice in healthcare provision, research and planning. At the same time, the wealth of data created from populationlevel $\mathrm{HbA} 1 \mathrm{C}$ screening also has the potential to allow us to better investigate, monitor and reduce the structurally embedded causes of population-level health inequities. 


\section{Footnotes}

$C D$ is funded through support from Alberta Innovates-Health Solutions Establishment and Incentive Grants (AI-HS to MR) and a University of Calgary Veterinary Medicine Postdoctoral Entrance Award. MR holds a Population Health Investigator award from Alberta Innovates-Health Solutions, which is funded by the Alberta Heritage Foundation for Medical Research Endowment. She also holds a New Investigator in Societal and Cultural Dimensions of Health Award from the Canadian Institutes for Health Research. WAR received no support from external funding for her contribution to this paper.

\section{References}

1. International Expert Committee. International Expert Committee Report on the role of the A1C assay in the diagnosis of diabetes. Diabetes Care2009;32:1327e34.

2. Skyler JS, Bergenstal R, Bonow RO, et al. Intensive glycemic control and the prevention of cardiovascular events: implications of the ACCORD, ADVANCE, and VA diabetes trials: a position statement of the American Diabetes Association and a Scientific Statement of the American College of Cardiology Foundation and the American Heart Association. Circulation2009;119:351e7.

3. DCCT Research Group.The effect of intensive treatment of diabetes on the development and progression of long-term complications in insulin-dependent diabetes mellitus. N Engl J Med1993;329:977e86.

4. UKPDS Group. Intensive blood-glucose control with sulphonylureas or insulin compared with conventional treatment and risk of complications in patients with type 2 diabetes (UKPDS 33). Lancet1998;352:837e53.

5. Selvin E,Steffes MW, Zhu H,et al. Glycated hemoglobin, diabetes, and cardiovascular risk in nondiabetic adults. N Engl J Med2010;362:800e11.

6. Feldman PJ, Steptoe A. Psychosocial and socioeconomic factors associated with glycated hemoglobin in nondiabetic middle-aged men and women. Health Psychol 2003;22:398e405.

7. Kelly SJ, Stedman J, Leonardi-Bee J. Is hemoglobin A1c level associated with measures of socio-economic status in non-diabetics after controlling for known explanatory factors? Stress Health2005;21:185e92.

8. Gillies $\mathrm{CL}$, Abrams KR, Lambert PC, et al. Pharmacological and lifestyle interventions to prevent or delay type 2 diabetes in people with impaired glucose tolerance: systematic review and meta-analysis. BMJ2007;334:299.

9. Crandall JP, Knowler WC, Kahn SE, et al. The prevention of type 2 diabetes. Nat Clin Pract End Met2008;4:382e93.

10. U.S. Preventive Services Task Force. Screening for type 2 diabetes mellitus in adults: U.S. Preventive Services Task Force recommendation statement. Ann Intern Med 2008;148:846e54.

11. Saudek CD, Herman WH, Sacks DB, et al. A new look at screening and diagnosing diabetes mellitus. J Clin Endocrinol Metab2008;93:2447e53.

12. Sheehy AM, Coursin DB, Gabbay RA. Back to Wilson and Jungner: 10 good reasons to screen for type 2 diabetes mellitus. Mayo Clin Proc2009;84:38e42. 
13. Selvin E,Steffes MW, Gregg E,et al. Performance of A1C for the classification and prediction of diabetes. Diabetes Care2011;34:84e9.

14. Bury M. Chronic illness as biographical disruption. Sociol Health Illn1982;4:167e82.

15. Department of Health. Second Report of the National Screening Committee. London: Stationery Office, 2000.

16. Eborall H, Griffin S, Prevost A, et al. Psychological impact of screening for type 2 diabetes: controlled trial and comparative study embedded in the ADDITION (Cambridge) randomised controlled trial. BMJ2007;335:486e9.

17. Paddison CAM, Eborall HC, Sutton S, et al. Are people with negative diabetes screening tests falsely reassured? Parallel group cohort study embedded in the ADDITION (Cambridge) randomised controlled trial. BMJ2009;339.

18. Mol A. The Logic of Care: Health and the Problem of Patient Choice. New York: Routledge, 2008.

19. Mann E, Prevost AT, Griffin S, et al. Impact of an informed choice invitation on uptake of screening for diabetes in primary care (DICISION): trial protocol. BMC Public Health2009;9:63.

20. Sargeant LA, Simmons RK, Barling RS, et al. Who attends a UK diabetes screening programme? Findings from the ADDITION-Cambridge study. Diabet Med 2010;27:995e1003.

21. Entwistle VA, Carter SM, Trevena L, et al. Communicating about screening. BMJ 2008;337:a1591.

22. Bodenheimer $\mathrm{T}$, Lorig $\mathrm{K}$, Holman $\mathrm{H}$,et al. Patient self-management of chronic disease in primary Care. JAMA2002;288:2469e75.

23. Brown AF, Ettner SL, Piette J, et al. socioeconomic position and health among persons with diabetes mellitus: a conceptual framework and review of the literature. Epidemiol Rev2004;26:63e77.

24. Glazier RH, Bajcar J, Kennie NR, et al. A systematic review of interventions to improve diabetes care in socially disadvantaged populations. Diabetes Care 2006;29:1675e88.

25. Diabetes Prevention Program Research Group.10-year follow-up of diabetes incidence and weight loss in the Diabetes Prevention Program Outcomes Study. Lancet2009;374:1677e86.

26. Ackerman R,Finch E, Brizendine E,et al. Translating the Diabetes Prevention Program into the Community: The DEPLOY Pilot Study. Am J Prev Med2008;35:357e63.

27. Rogers WA. Evidence based medicine and justice: a framework for looking at the impact of EBM upon vulnerable or disadvantaged groups. JMedEthics2004;30:141e5.

28. Beauchamp T. Moral foundations. In: Coughlin S, Beauchamp T, eds. Ethics and Epidemiology. New York: Oxford University Press, 1996:24e52.

29. Cohen MZ,Tripp-Reimer T, Smith C,et al. Explanatory models of diabetes: patient practitioner variation. Soc Sci Med 1994;38:59e66.

30. Marmot M. Achieving health equity: from root causes to fair outcomes. Lancet 2007;370:1153e63.

31. Brock DW. Priority to the Worse off in Health-Care Resource Prioritization. Medicine and Social Justice: Essays on the Distribution of Health Care. Oxford: Oxford University Press, 2002. 
32. Long J, Field S, Armstrong K, et al. Social capital and glucose control. J Community Health2010;35:519e26.

33. Rose G. Strategy of prevention: lessons from cardiovascular disease. BMJ 1981;282:1847e51.

34. Holt-Lunstad J,Smith TB, Layton JB. Social relationships and mortality risk: a metaanalytic review. PLoS Med2010;7:e1000316.

35. Maitra S. Can patient self-management explain the health gradient? Goldman and Smith's "Can patient self-management help explain the SES health gradient?" (2002) revisited. Soc Sci Med2010;70:802e12. 